\title{
Determining the Equivalent Conicity for Railway Wheelset Maintenance with Deep Ensembles
}

\author{
Alexandre Trilla ${ }^{1}$, and Xavier Cabré ${ }^{2}$ \\ 1,2 ALSTOM, Santa Perpètua de la Mogoda, Barcelona, 08130, Spain \\ alexandre.trilla@alstomgroup.com \\ francesc-xavier.cabre@alstomgroup.com
}

\begin{abstract}
The Equivalent Conicity is an indirect measure that is indicative of the amount of dynamic instability for a given wheelset. Therefore, it cannot be acquired directly from a physical component, but needs to be determined with related physical measures. The interaction between the conical wheel profiles, their diameter, the track profile, and its gauge, produces bogie hunting oscillations that may incur the risk of derailment if they are allowed to reach large magnitude values, which are attained as the wheel treads degrade with use. This is especially critical for high-speed rail environments. However, the Equivalent Conicity figure helps to quantify this effect and thus drive an effective wheel reprofiling maintenance schedule. This article conducts an evaluation of the Equivalent Conicity algorithm for two trains of the British Rail Class 390 fleet (Pendolino). It assesses the standard calculation approach based on the differential equations, and develops a data-driven approach based on a deep ensemble of features with neural networks. The corresponding values provided by Delta Rail are used as the ground truth. The results of the analysis prove that this method meets the requirements of the maintenance staff and thus yields a new avenue for business improvement through the application of the condition maintenance approach for wheelsets.
\end{abstract}

\section{INTRODUCTION}

The wheel-rail interface is fundamental to explain the dynamic running behaviour of a railway vehicle. In this sense, the Equivalent Conicity (EC) is an indirect parameter (i.e., calculated) for the wheelset that facilitates the analysis of this interface (Thomsen, P. G. and True, H., 2010). It may determine possible vibrations and irregularities in vehicle dynamics as well as the critical speed of railway vehicles (Gerlici, Domin, Cherniak, \& Lack, 2018). The EC also describes their motion pattern, also known as the hunting oscillation, which

\footnotetext{
Alexandre Trilla et al. This is an open-access article distributed under the terms of the Creative Commons Attribution 3.0 United States License, which permits unrestricted use, distribution, and reproduction in any medium, provided the original author and source are credited.
}

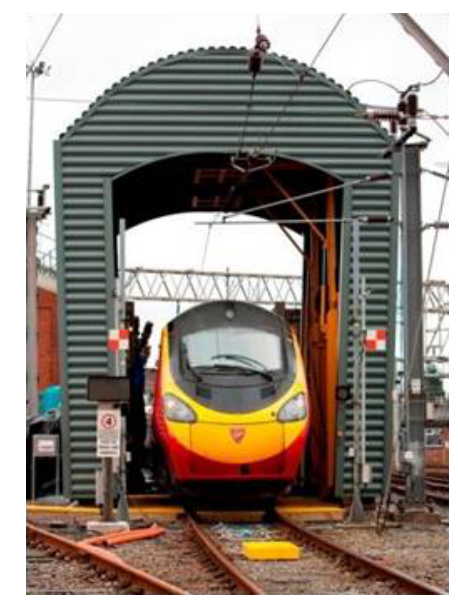

Figure 1. ALSTOM TrainScanner deployment at the Manchester Traincare Centre.

in turn permits drawing conclusions on the driving comfort and safety (Polach, 2010). A strong conicity characterises a contact capable of self-orienting the axle, but it risks making the bogie unstable. There are two standard techniques to approach this calculation: one the is based on the integration of the nonlinear equation for wheelset dynamics, and another one that is based on the linear regression of the rolling radius function (BS, 2011).

From a maintenance perspective, this dynamic interaction is useful to assess the condition of the wheelsets, which may then be used to drive the reprofiling actions. In this regard, ALSTOM has developed the TrainScanner, see Figure 1, which is a train monitoring system that is aimed at optimising the maintenance of brake pads, pantograph carbon strips, and wheelsets, through the deployment of the PHM methodology and its associated techniques. TrainScanner integrates a series of acquisition subsystems with lasers and 3D cameras that capture the related measures as a train traverses its portal. Then, it automatically conducts the processing and analysis of the collected data, and finally it triggers alarms and issues reports to the maintenance staff. This work is par- 
ticularly focused on the wheel profile data and their use for extracting the $\mathrm{EC}$ value.

This article is organised as follows: Section 2 describes the analysis procedure that has been followed, including the description of the data, the EC evaluation technique based on the wheelset dynamics, their management, adjustment, and refinement with a deep ensemble, along with preliminary results. Section 3 discusses the overall results and the limitations of the approach, and Section 4 concludes the manuscript and reflects on its impact to the current maintenance plan.

\section{Methods AND Results}

This section describes the sequential process that has been followed in order to obtain a robust EC calculation procedure. Thus, the development is incremental and preliminary results are provided.

\subsection{Wheel Profile Data}

The dataset consists of a compilation of wheel profiles captured with MiniProf for two trains, with 72 wheelsets each, on October 2016 and January 2017. Nevertheless, there are three wheelsets that do not show consistent data, either by missing one of the wheels, or by showing a bad profile acquisition. We attribute this issues to improper handling of the tool (we trust that MiniProf is adequate for acquiring the profiles).

Additionally, the remaining 141 wheelsets also show inconsistencies with regard to the wheel diameter values. These are expected to be between $920 \mathrm{~mm}$ when new, and $850 \mathrm{~mm}$ when worn-out. It happens that 58 wheels are strictly out of this range (some assets even display diameters around $450 \mathrm{~mm}$ and $1200 \mathrm{~mm}$ ), revealing that the acquired data is unreliable. Anyhow, all these data inconsistencies at the wheelset level do have Delta Rail EC reference values.

\subsection{Calculation Based on Wheelset Dynamics}

We developed an initial approach toward the calculation of the EC with the wheel profile data following the method defined in the BS 15302 standard (BS, 2011), which is based on the differential equations that define the wheelset dynamics. However, this algorithm only works if the two wheel profiles for a given wheelset are identical. Thus, in order to make use of it, two virtual wheelsets are constructed with a duplicate of the two available wheel profiles $\left(w_{1}\right.$ and $\left.w_{2}\right)$, and the maximum $\mathrm{EC}$ value is determined (taken for the most critical situation), see Eq. (1).

$$
E C\left(w_{1}, w_{2}\right)=\max \left(E C\left(w_{1}, w_{1}\right), E C\left(w_{2}, w_{2}\right)\right)
$$

Experimentally, our implementation also shows to be rather invariant to contextual parameters like the track gauge. Thus,

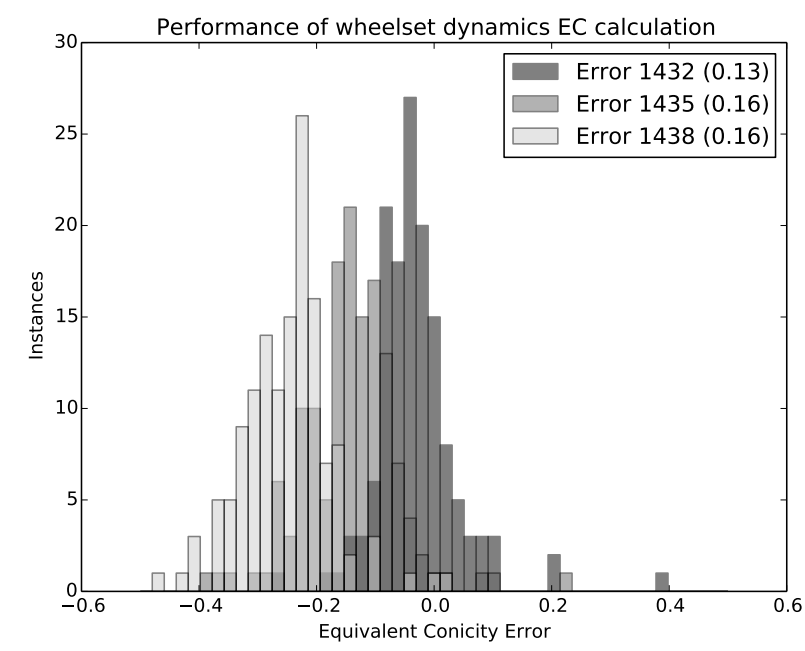

Figure 2. EC error with wheelset dynamics. In brackets, its uncertainty.

it is expected to display large bias errors when different gauges are evaluated, namely $1432 \mathrm{~mm}, 1435 \mathrm{~mm}$ and $1438 \mathrm{~mm}$.

The difference between the ground truth (Delta Rail) and the wheelset dynamics procedure is computed (it represents the error of the approach) and shown in Figure 2. The error distributions are centred around a mean point that is $\mu_{1432}=$ $-0.03, \mu_{1435}=-0.14$, and $\mu_{1438}=-0.25$. This bias can be easily corrected with a constant term of equivalent magnitude, though. The actual uncertainty of the calculation is given by the maximum deviation, which stretches from 0.13 to 0.16 .

\subsubsection{Estimation of Uncertainty}

The specific statistical terms of "accuracy" and "precision" are related with the difference between two values, both in terms of bias and variance error. Its bias, also known as trueness (ISO, 1994), is of little importance in this work to evaluate the effectiveness of a predictive technique, because it can be easily corrected if it is known (or experimentally estimated) in advance, which is a side objective of the techniques presented in this paper (the main use of bias is for detecting model underfitting). However, the variability of the error has a random nature, and this is the main driver of the calculation performance: its uncertainty is assumed to represent the expected maximum variability of the error, for a confidence interval of $95 \%$.

The goal of the following sections is to apply data science techniques in order to learn the relation between input wheelset data and output EC values, and derive new EC functions that take advantage of this learnt knowledge. It must be stated that whenever the uncertainty of a given approach 


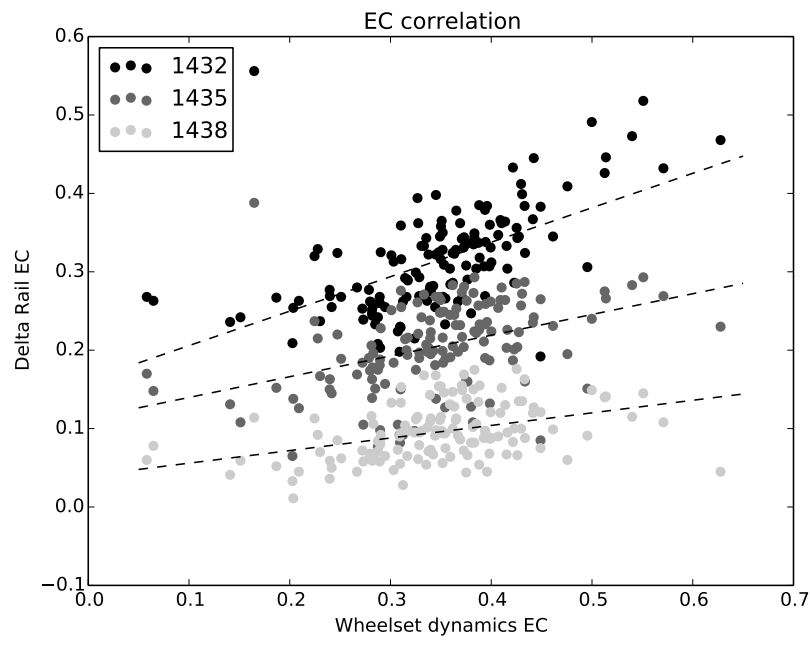

Figure 3. Linear correlation between the wheelset dynamics EC results and Delta Rail.

is evaluated, a statistical procedure known as the Leave-OneOut Cross-Validation (LOO-CV) ("Encyclopedia of Machine Learning and Data Mining", 2010) is used. This validation process uses the complete set of data for training but one instance, which is then used for testing. The procedure is repeated until all instances are tested. This ensures that all the predictions will be produced with data that is not used for training, thus providing a reliable estimation of the actual performance (thus, the size of the sample is of 141 instances).

\subsection{Linear Correlation Correction}

In order to gain more insight into the relation between the calculated EC value and its actual value, Figure 3 shows their linear correlation.

It is clear that there exists a relation between the wheelset dynamics results and the ground truth provided by Delta Rail taking the track gauge into account. Following the LOO-CV procedure, the linear regression method is used to model this mapping. After scoring the new error results with the corrected EC values, the results are shown in Figure 4.

It can be seen that after the linear mapping is applied, the accuracy of the prediction drops for all track gauges. This represents an absolute EC uncertainty improvement of 0.09 (56\%) in the best cases. This work considers this result to be its baseline. Additionally, this result shows that the wheelset dynamics based calculation may be taken for an "inaccurate and imprecise sensor of EC", which can be easily compensated with an offset and gain error correction using the linear mapping. The next section explores a way to further augment the representation space of the wheelset features.

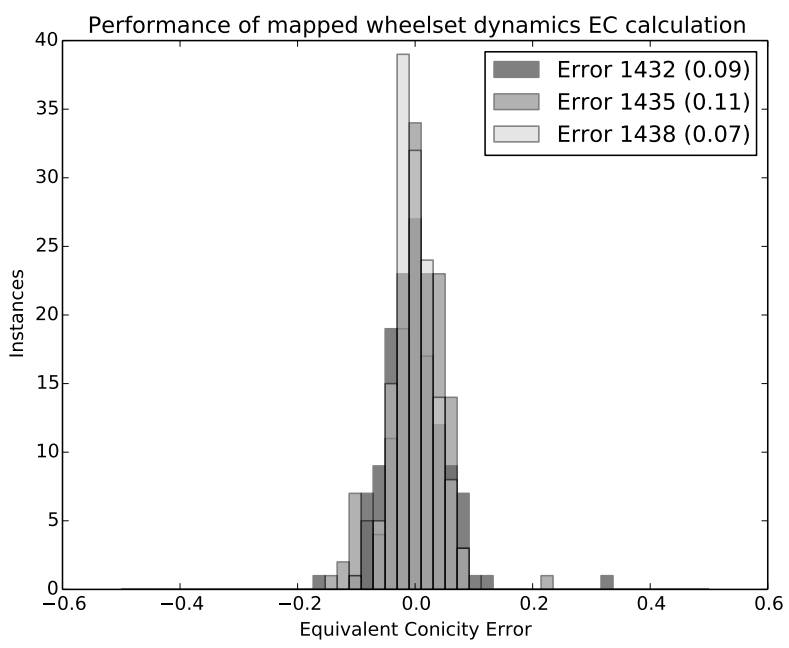

Figure 4. Mapped wheelset dynamics EC results. In brackets, the estimated uncertainty.

\subsection{Wheel Tread Slope}

This section processes the wheel profile directly in order to extract the slope of the tread, see Figure 5, as a means to expand the feature space. The GM/RT2466 standard (RSSB, 2010) states that the central point of the tread is to be located at $70 \mathrm{~mm}$ with respect to the flange back. This study considers a tread width of $20 \mathrm{~mm}$ around its centre in order to extract the slope. This region should begin at the end of the flange foot, and should not be affected by the chamfer.

Figure 6 shows the correlation between the wheel tread slopes and the reference EC provided by Delta Rail. Note that this relational analysis is only conducted at the wheel level, whereas the EC is actually related to the wheelset. Thus, the two wheel slopes have an impact on the EC value. In consequence, there might be wheel slope combinations that yield a clearer relation. In addition, it can observed that there seems to be a non-linear effect with an inflection around the -0.5 value. Whether if the tread slope will add knowledge to the EC calculation will be determined in the next section.

\subsection{Deep Ensemble}

Deep Learning are a type of complex computational models composed of multiple non-linear processing units stacked into layers which learn representations of data with multiple levels of abstraction (LeCun, Y. and Bengio, Y. and Hinton, G., 2015). Their greatest success has been driven by their good performance with discovering intricate structure in large datasets of raw signals. They display a neural network architecture (Hertz, Krogh, \& Palmer, 1991), and share many traits with them. Like neural networks, they exploit the connectionist learning approach, where the links among the units are weighted in order to accomplish a specific task. 


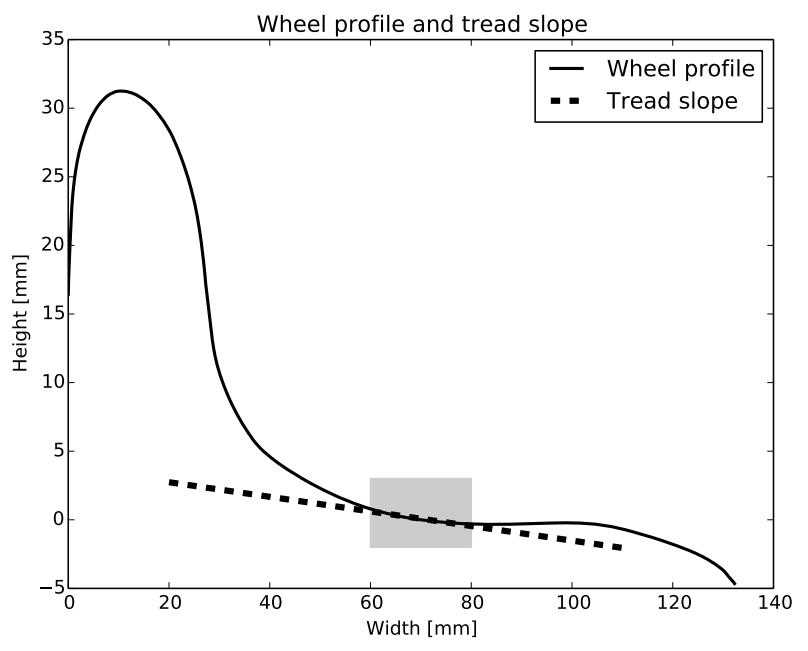

Figure 5. Wheel profile and tread slope.

In this regard, one of their greatest advantages is their ability to seamlessly integrate data from different sources. Figure 7 shows a deep learning framework that integrates two neural networks, blending the wheelset dynamics calculation with the wheel tread slopes, thus creating an ensemble of features.

The stacking of the neurons into layers and their feed-forward arrangement from left to right is known as multilayer perceptron, and it is a very practical architecture for solving generalpurpose problems (Bishop, C. M., 2006; Duda, Hart, \& Stork, 2000). The range of the input values needs to be normalised around a similar magnitude to guarantee an effective learning convergence, so the wheel tread slope is multiplied by 10. The non-linear smoothing function for all the units is set to be the logistic sigmoid. Therefore, the maximum output value of the network (i.e., an EC value of 0.6) is guaranteed not to saturate the unit.

\subsubsection{Granularity Goals}

The two neural networks are trained sequentially with stochastic gradient descent using backpropagation. However, their learning parameters differ in order to attain different levels of detail:

- Coarse-grained: the goal is to calculate an approximation to the corrected EC value and maximise the generalisation of the network. This is attained with a fast learning rate of 0.5 that is checked to avoid cost overshooting, and a maximum number of training dataset iterations of 300 to consider early stopping and thus prevent overfitting.

- Fine-grained: the goal is to calculate a refined EC value and maximise the specialisation of the network. This is attained with a slow learning rate of 0.1 , and a greater number of training dataset iterations up to 10000.

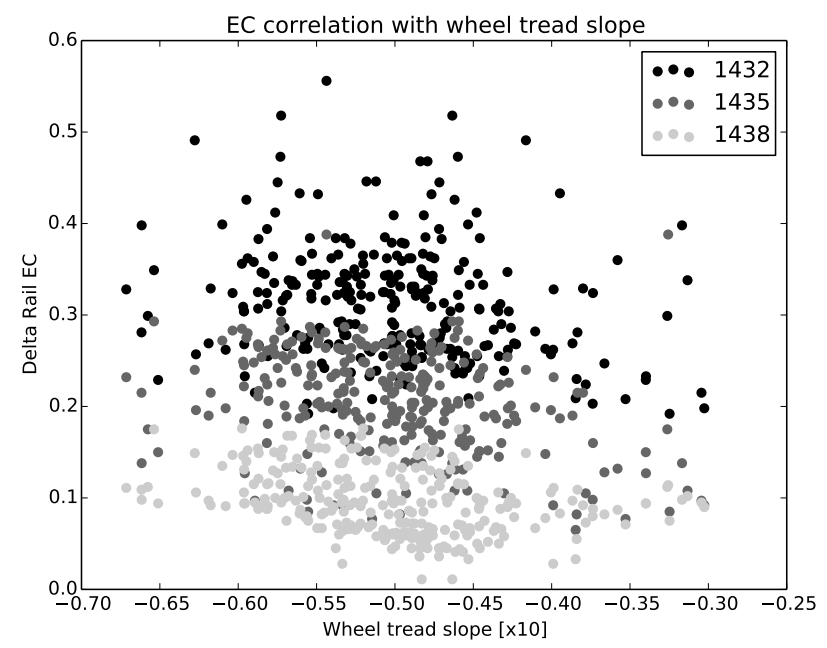

Figure 6. Correlation between the wheel tread slopes and Delta Rail EC.

The challenge with a multilayer neural network is to match its expressiveness, which is related to the number of hidden units $H$, with the complexity of the data. The more weights is has (for every hidden unit, 7 new weights are added to the network), the more data idiosyncrasies is it able to learn, at the risk of overfitting. Figure 8 shows the results of this study for the coarse-grained sub-network. It can be seen that the performance score displays an overall minimum when the size of the hidden layer is of 4 units. This configuration is maintained for the fine-grained sub-network, and the error distribution for the complete deep ensemble is shown in Figure 9, where it can be seen that the final system outperforms the previous approaches and reaches a minimum EC uncertainty score of 0.05 for the $1438 \mathrm{~mm}$ gauge.

\section{DISCUSSION}

The use of MiniProf data (instead of TrainScanner data) to validate the performance of the EC algorithm requires doing modifications which may introduce an additional uncertainty, especially because the algorithms need to be tweaked in order to accommodate signals from different sources. In addition, the available MiniProf data shows acquisition issues (attributed to tool mishandling), which invalidate the calculation of the EC values for the related wheelsets taking the BS 15302 standard into account. However, Delta Rail does provide results for these wheelsets, which proves that the reference calculation has been conducted differently (not as is strictly described in the regarded standard, which requires the profiles for the two wheels, along with their diameters). Therefore, other approaches to the EC calculation may need to be considered.

The algorithm based on the wheelset dynamics is invariable to track gauge, despite passing the quality test with a benchmark 


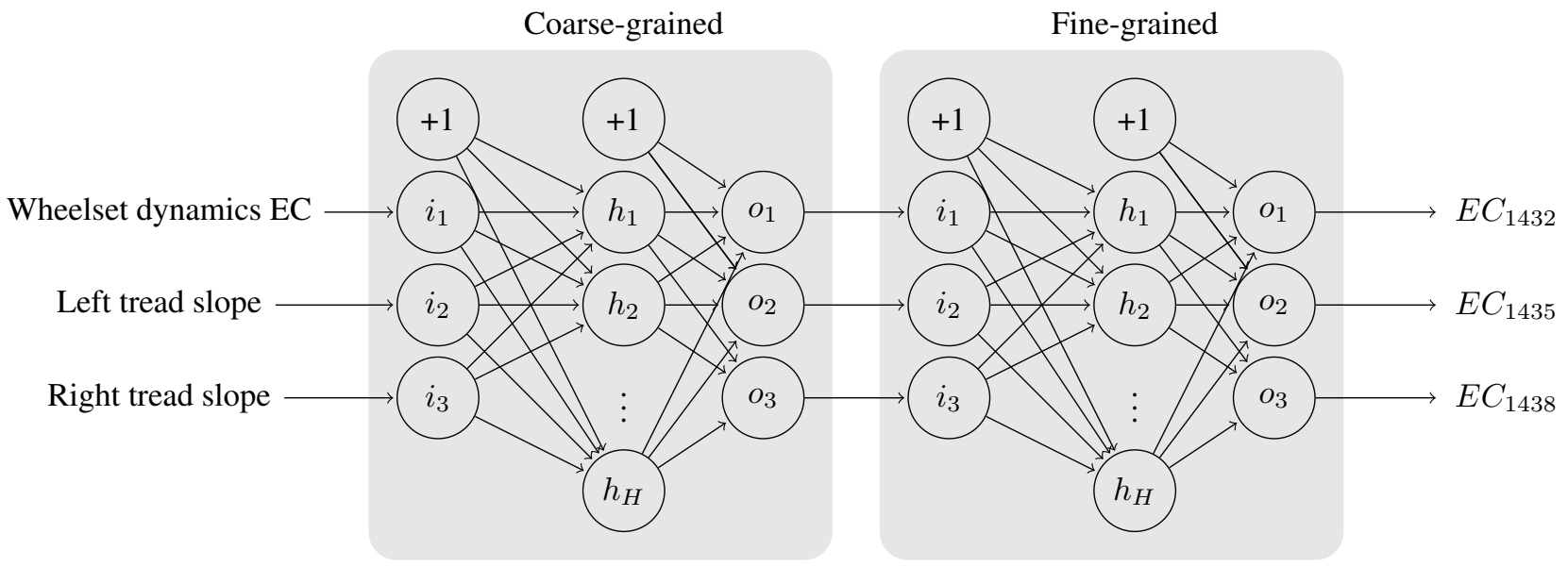

Figure 7. Deep learning feature ensemble for refined EC calculation. Neural networks trained with different granularity goals are connected.

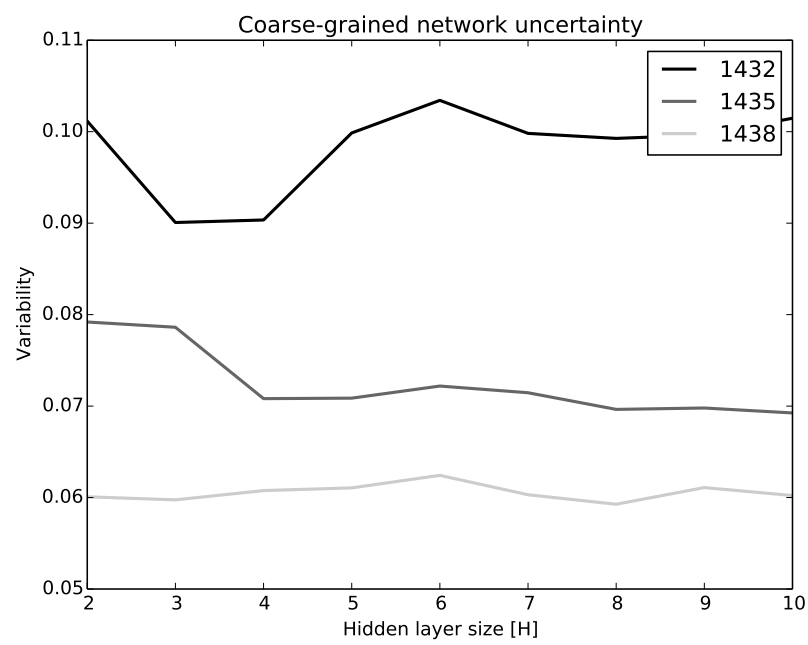

Figure 8. Coarse-grained neural network performance with respect to the size of its hidden layer.

calculation using defined reference profiles with errors, as is required by the standard. This might be attributed to numeric precision or algorithm conditioning, being the latter the most probable cause. Nevertheless, this approach proves to be inadequate for real wheel profile data. However, it provides a rough approach that can be used (with further statistical processing using a deep neural structure) to produce an EC value that is useful for maintenance. In this sense, the inclusion of the wheel tread slopes through a deep ensemble has permitted exploiting their non-linear correlation to gradually reduce the uncertainty of the calculation.

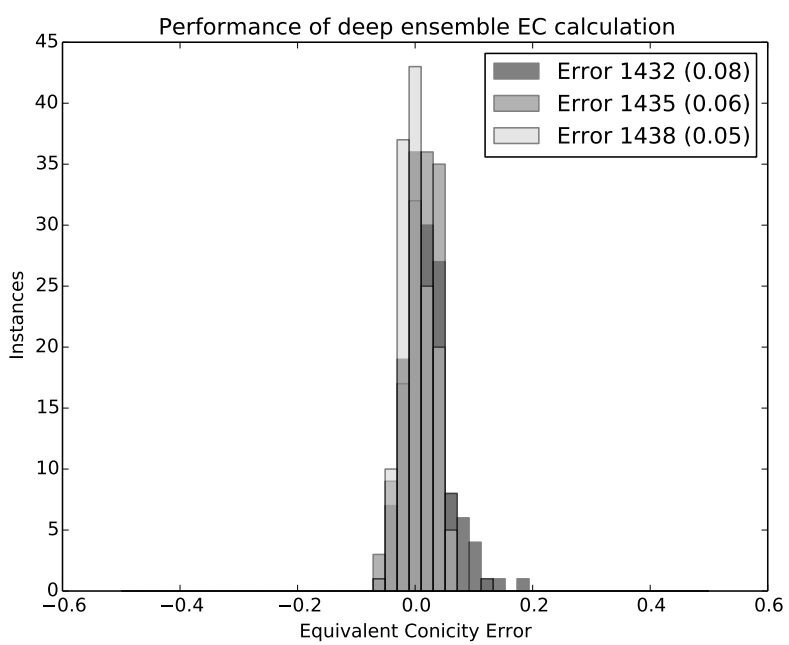

Figure 9. Histogram of the refined EC calculation with the deep ensemble. In brackets, the estimated uncertainty.

\section{Conclusion}

At present, the reprofiling maintenance criterion for the Class 390 wheelsets is based on a periodic mileage value. This is an evident ineffective approach because it does not take into account the actual degree of wear of the treads, which is definitely impacted by the seasonal weather, the driving style, the condition of the track, etc. The EC calculation, instead, provides an indirect indicator for its condition through the wheelset instability characteristic, which is regarded as a more accurate approach to its maintenance.

This article presents the most sophisticated technique for TrainScanner EC calculation, which is based on a deep neural 
network ensemble that blends a preliminary calculation based on wheelset dynamics with the wheel tread slope features. It yields a performance around 0.05 at the asset level, which complies to the maintenance requirements. Its effectiveness has proven to enhance to the former approaches solely based on the individual wheelset dynamics EC calculation, including the linear correction applied a posteriori.

The future work that is currently envisaged may further deal with data idiosyncrasies in order to add more robustness to the method, dealing with the data that lies out of the confidence interval. Alternatively, we also expect to explore other learning paradigms and seek the complementary characteristics that may help the current approach thrive and further push the effectiveness boundary.

\section{REFERENCES}

Bishop, C. M. (Ed.). (2006). Pattern Recognition and Machine Learning (Information Science and Statistics). Springer-Verlag New York, Inc.

BS. (2011). Railway applications - Method for determining the equivalent conicity. British Stan$\operatorname{dard}(15302: 2008+\mathrm{A} 1: 2010)$.

Duda, R. O., Hart, P. E., \& Stork, D. G. (Eds.). (2000). Pattern Classification. Wiley-Interscience.

Encyclopedia of Machine Learning and Data Mining. (2010). In Sammut, C. and Webb, G. I. (Ed.), . Springer US.

Gerlici, J., Domin, R., Cherniak, G., \& Lack, T. (2018). Calculated estimation of railway wheels equivalent conicity influence on critical speed of railway passenger car. MATEC Web of Conferences, 157, 1-7.

Hertz, J., Krogh, A., \& Palmer, R. G. (Eds.). (1991). Introduction to the theory of neural computation. AddisonWesley Longman Publishing Co., Inc.
ISO. (1994). Accuracy (trueness and precision) of measurement methods and results - Part 1: General principles and definitions (Tech. Rep. No. 5725-1:1994). International Organization for Standardization.

LeCun, Y. and Bengio, Y. and Hinton, G. (2015). Deep Learning. Nature, 521, 436-444.

Polach, O. (2010). Application of Nonlinear Stability Analysis in Railway Vehicle Industry. In Non-Smooth Problems in Vehicle Systems Dynamics.

RSSB. (2010). Railway Wheelsets (Tech. Rep. No. GM/RT2466). Railway Group Standards.

Thomsen, P. G. and True, H. (Ed.). (2010). Non-Smooth Problems in Vehicle Systems Dynamics. Springer US.

\section{BIOGRAPHIES}

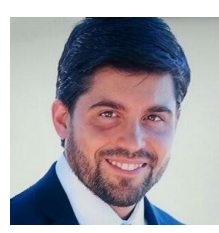

Alexandre Trilla graduated from LaSalle University of Barcelona with a M.Sc. in Electronics and Telecommunications Engineering in 2008, and a M.Sc. in IT Management in 2010. He has an academic research background in spoken language processing, and an industrial research background in PHM. He has authored several publications in scientific conferences and journals (IEEE Transactions on Audio, Speech, and Language Processing, Chemical Engineering Transactions, and the Journal of Rail and Rapid Transit). At present, he is a data scientist working on the deployment of PHM to the railway environment.

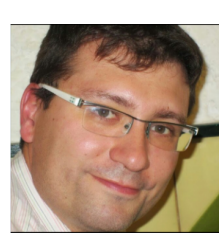

Xavier Cabré was born in Barcelona. He graduated from LaSalle University of Barcelona with a M.Sc. in Electronics and Telecommunications Engineering in 1998. He has an industrial research background in high-technology environments. In 2015, he co-authored a patent in image processing and computer vision for the detection of railway components. At present he is the Project/Program manager of TrainScanner. 